\title{
Eficiência Reprodutiva e Produtiva em Rebanhos Leiteiros Comerciais Monitorados por Sistema Informatizado 1
}

\author{
Selma de Fátima Grossi ${ }^{2}$, Maria Armênia Ramalho de Freitas ${ }^{3}$
}

\begin{abstract}
RESUMO - O estudo foi conduzido com os objetivos de analisar o desempenho produtivo e algumas medidas de eficiência reprodutiva de 1877 vacas provenientes de quatro grupos genéticos Holandês - Zebu e estimar os parâmetros genéticos pertinentes. Os animais pertenciam a três rebanhos comerciais que foram monitorados pelo Sistema Computacional de Informação DAISY (The Dairy Information System), durante período de 1989 a 1998. Para consistência dos dados, formação dos arquivos e análises preliminares foram usados os procedimentos disponíveis no SAS (Statistical Analysis System), enquanto os componentes de (co)variância foram estimados pelo método de máxima verossimilhança restrita livre de derivada (MTDFREML), sob um modelo animal. Os efeitos de grupo genético e ano do parto foram significativos sobre a idade da vaca ao primeiro parto (IPC), intervalo parto - primeiro serviço (PP1S), duração da lactação(DL), produção total de leite (PT) e produção de leite por dia de intervalo de parto (PLIEP). As estimativas de herdabilidade $\left(\mathrm{h}^{2}\right)$ para as características reprodutivas ficaram próximas de zero, evidenciando grande dependência do manejo oferecido aos rebanhos, enquanto o valor de 0,28 para a $\mathrm{h}^{2}$ da PT mostrou variância genética aditiva média. A correlação genética entre PT e DL de 0,81 pode ser considerada de alta magnitude.
\end{abstract}

Palavras-chave: aspectos genéticos e de ambiente, gado de leite, vacas cruzadas

\section{Reproductive and Productive Efficiency in Commercial Dairy Herds Monitored by Information System}

\begin{abstract}
The objectives of this work were to evaluate the productive and reproductive efficiencies and also the genetics parameters for traits considered, using data from 1877 crossbreed cows (Holstein x Zebu) from four genetics groups. The cows belonged to three commercial dairy herds monitored from 1989 to 1998 by the Dairy Information System (DAISY). The data were processed using the procedure available in SAS (Statistical Analysis System), while genetic parameters on productive and reproductive traits were estimated by the multiple trait derivative-free restricted maximum likelihood method (MTDFREML). Effects of genetic group and calving year were significant for age at first calving (AFC), interval from calving to first service (ICFS), lactation length (LL), milk production (MY) and milk production per day of calving interval (MYCI). The estimates of heritability $\left(\mathrm{h}^{2}\right)$ for all reproductive traits were near zero in agreement with others studies indicating that these traits are mostly determined by management practices. The $\mathrm{h}^{2}$ estimates for milk yield (MY) were 0.28 , this means that the additive genetic variance is middle. The genetic correlations $\left(\mathrm{r}_{\mathrm{g}}\right)$ between MY and LL of 0.81 could be considered high.
\end{abstract}

Key Words: genetic parameters, dairy cattle, crossbreed cows

\section{Introdução}

Na região Sudeste do Brasil, é conhecida a predominância do gado mestiço leiteiro, oriundo de vários sistemas de cruzamento entre as raças européias especializadas com as raças zebuínas. Essa prática tem permitido a exploração da heterose e, conseqüentemente, a obtenção de animais mais produtivos e adaptados a cada sistema de produção. O aumento da produtividade desses sistemas, mediante o melhoramento dos índices técnicos e econômicos, é funda- mental para nossa pecuária leiteira tanto para a sobrevivência dos produtores na atividade quanto para oferecerem produto mais competitivo e de melhor qualidade ao mercado.

A produção de leite e os aspectos reprodutivos são processos determinantes nessa eficiência de produção dos rebanhos leiteiros pelos seus reflexos diretos na produtividade e rentabilidade (Madalena et al., 1996; Freitas et al., 1996; Ferreira \& Madalena, 1997; Freitas et al., 1997). A importância do estudo das características reprodutivas e produtivas assim

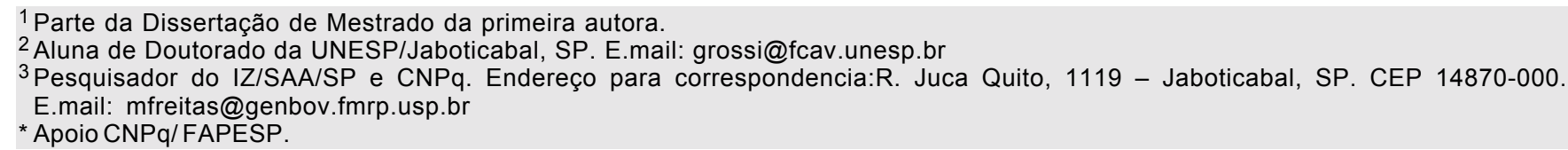


como os aspectos fenotípicos e genéticos, envolvidos nas mesmas, estão bem destacados pela literatura (Monardes et al., 1995; Prado et al., 1995; Ferreira \& Ferreira, 1998; e Zambianchi et al., 1997). Assim, este estudo teve como objetivos analisar o desempenho produtivo e reprodutivo de animais oriundos de quatro grupos genéticos Holandês - Zebu e estimar os parâmetros genéticos pertinentes. Estudos desse tipo podem fornecer elementos que certamente irão contribuir na definição de estratégias de seleção desses grupamentos genéticos, além de auxiliar o produtor de leite nas tomadas de decisões pertinentes ao manejo e administração da sua propriedade, visando o melhoramento da eficiência de produção.

\section{Material e Métodos}

Os registros de desempenho reprodutivos e produtivos de 1877 vacas Holandês-Zebu (1/2HZ, 5/8H, $3 / 4 \mathrm{H}, 7 / 8 \mathrm{H}$ ), pertencentes a três rebanhos leiteiros comerciais, mantidos na região Sudeste do Brasil entre 1989 e 1998, foram analisados. As propriedades adotavam medidas de alimentação e manejo satisfatórias para o nível de produção dos seus animais, registravam sistematicamente os dados zootécnicos referentes aos aspectos reprodutivos e da produção de leite, os quais eram processados e analisados pelo sistema informatizado DAISY (Dairy Information System). Dos relatórios técnicos e de rotina emitidos mensalmente pelo sistema, foram escolhidas as características a serem analisadas neste estudo quais sejam: idade à primeira cria (IPC); intervalo parto - primeiro serviço (PP1S), período de serviço (PS), intervalo de partos (IEP), produção de leite (PT), duração da lactação (DL) e a produção de leite por dia de intervalo de parto (PLIEP).

Para consistência dos dados, formação dos arquivos e análises preliminares foram utilizados os procedimentos disponíveis no programa SAS, (SAS 1992). Nessas análises os seguintes fatores de variação foram considerados: rebanho, ano do parto, grupo genético, estação do parto e ordem do parto, com a finalidade de observar o comportamento de cada uma dessas variáveis isoladamente sobre as características em estudo. Nas estimativas dos parâmetros genéticos, o modelo de análise incluiu os efeitos de grupo genético, grupo contemporâneo constituído de rebanho, ano e estação do parto e idade da vaca ao parto como única (co)variável no modelo, além do efeito aleatório de animal. Para tanto os componentes de (co)variância foram estimados pelo método da máxima verossimilhança restrita, através do software MTDFREML (Multiple trait Derivative-Free Restricted Maximum Likehood), sob um modelo animal, descrito por Boldman et al. (1995), cuja forma matricial foi assim representada:

$$
\mathrm{Y}=\mathrm{Xb}+\mathrm{Zu}+\mathrm{Wp}+\mathrm{e}
$$

em que: $Y=$ vetor de informações; $b=$ vetor de efeitos fixos; $X=$ matriz de incidência que associa $\beta \operatorname{com} X$; $\mathrm{u}=$ vetor dos efeitos aleatórios [genético aditivo]; $\mathrm{Z}=$ matriz de incidência, que associa elementos de u com $\mathrm{Y} ; \mathrm{p}=$ vetor dos efeitos aleatórios [ambiente permanente]; $\mathrm{W}=$ matriz de incidência para os efeitos de ambiente permanente; $\mathrm{e}=$ vetor dos efeitos aleatórios residuais.

Pressuposições: $\mathrm{V}(\mathrm{v})=\mathrm{G}=\mathrm{A} \sigma^{2} ; \mathrm{V}(\mathrm{p})=\mathrm{P}=\mathrm{I} \sigma_{\mathrm{p}}^{2}$; $\mathrm{V}(\mathrm{e})=\mathrm{R}=\mathrm{I} \sigma_{\mathrm{e}}^{2} ; \operatorname{Cov}\left(v, \mathrm{e}^{\prime}\right)=0 ; \operatorname{Cov}(\mathrm{p}, \mathrm{e})=0 ; \operatorname{Cov}(\mathrm{e} . \mathrm{p})=0$

e pode ser representado como:

$$
\mathrm{E}\left[\begin{array}{l}
\mathrm{Y} \\
\mathrm{p} \\
\mathrm{v} \\
\mathrm{e}
\end{array}\right]=\left[\begin{array}{c}
\mathrm{X} \beta \\
0 \\
0 \\
0
\end{array}\right]
$$

Foram realizadas análises uni e bivariada, para obtenção dos componentes de (co)variância das características intervalo de partos e produção total de leite, duração da lactação e produção de leite por dia de intervalo de partos.

\section{Resultados e Discussão}

A média observada para à idade a primeira cria foi de 32 meses, com desvio padrão de 5 meses, valor que pode ser considerado satisfatório, situando-se próximo ao desejável para as condições brasileiras. Sabe-se que essa característica é bastante influenciada pelo manejo que o produtor oferece ao seu rebanho e depende, principalmente, da alimentação e manejo oferecidos aos animais nos primeiros meses de vida, possibilitando desenvolvimento satisfatório nas diversas etapas do seu crescimento. Outros fatores também atuam nesse processo como a verificação correta do cio e habilidade do inseminador, entre outros. A análise de variância mostrou que os efeitos de rebanho, grupo genético, ano e estação do parto foram significativos $(\mathrm{p}<0,01)$ sobre essa característica conforme se pode observar na Tabela 1.

Verificou-se ainda que o efeito de rebanho foi uma importante fonte de variação sobre as demais características reprodutivas analisadas $(\mathrm{p}<0,01)$. 
Tabela 1 - Síntese da análise de variância das características reprodutivas

Table 1 - Analyses of variance of reproductive traits

\begin{tabular}{|c|c|c|c|c|}
\hline $\begin{array}{l}\text { Variável } \\
\text { Variable }\end{array}$ & $\begin{array}{l}\operatorname{IPC}(1) \\
A F C(1)\end{array}$ & $\begin{array}{l}\operatorname{PP} 1 \mathrm{~S}(2) \\
I C F S(2)\end{array}$ & $\begin{array}{l}\mathrm{PS}(3) \\
D O(3)\end{array}$ & $\begin{array}{l}\operatorname{IEP}(4) \\
C I(4)\end{array}$ \\
\hline Rebanho & $531,4 * *$ & $17201,2 * *$ & $59492,9 * *$ & $60854,1 * *$ \\
\hline $\begin{array}{l}\text { Herd } \\
\text { Ano do parto }\end{array}$ & $716,4 * *$ & $18756,9 * *$ & $7816,6^{*}$ & $5129,8^{\mathrm{NS}}$ \\
\hline $\begin{array}{l}\text { Year of calving } \\
\text { Grupo genético }\end{array}$ & $237,9 * *$ & $6015,2 *$ & $10947,7^{*}$ & $4778,3^{\mathrm{NS}}$ \\
\hline $\begin{array}{l}\text { Genetic group } \\
\text { Estação do parto } \\
\text { Season of calving }\end{array}$ & $130,9 * *$ & $12490,7 * *$ & $19906,2 * *$ & $2531,0^{\mathrm{NS}}$ \\
\hline Ordem do parto & - & $3597,9^{\mathrm{NS}}$ & $3799,3^{\mathrm{NS}}$ & $13594,8^{*}$ \\
\hline
\end{tabular}

Calving order

NS = não significativo; ${ }^{* *}(p<0,01) ;{ }^{*}(p<0,05)$

$(1)$ = idade à primeira cria; $(2)=$ período parto-primeiro serviço; $(3)$ = período de serviço; $(4)=$ intervalo de partos.

$N S=$ not significant; ${ }^{* *}(p<.01) ;{ }^{*}(p<.05)$.

(1) = age at first calving; (2) = interval from calving to first service; (3) = days open; (4) = calving interval.

Enquanto grupo genético, ano e estação do parto foram significativos apenas para PP1S e PS, evidenciando o comportamento biológico diferente de cada grupamento. A média geral para o intervalo de partos foi de 392,7 dias com desvio padrão de 71 dias, valor bastante razoável para as condições brasileiras e em especial para vacas cruzadas (Holandês-Zebu). Uma das hipóteses para explicar valores próximos a 400 dias para o IEP é atribuída ao produtor que, às vezes, por conveniência, retarda a inseminação de animais que atingem níveis altos de produção de leite no início da lactação, confirmando que as variações que ocorrem nessa característica estão relacionadas muito mais aos efeitos de ambiente que propriamente aos de origem genética. As médias estimadas e respectivos erros padrão para o intervalo entre o parto e o primeiro serviço para os quatro grupos genéticos estudados estão apresenta-

Tabela 2 - Médias estimadas, erros-padrão (EP) e número de observações $(\mathrm{N})$ para o intervalo do parto ao primeiro serviço (dias), de acordo com o grupo genético

Table 2 - Estimated means, standard error (SE) and number of observations for calving interval to first service (days), according to genetic group

\begin{tabular}{lccc}
\hline Grupo genético & $\mathrm{N}$ & Média & $\mathrm{EP}$ \\
Genetic group & $N$ & Mean & $S D$ \\
\hline 1 & 646 & 81,9 & $\pm 1,64$ \\
3 & 570 & 94,8 & $\pm 1,75$ \\
5 & 105 & 109,5 & $\pm 4,07$ \\
7 & 104 & 84,5 & $\pm 4,09$ \\
\hline
\end{tabular}

$1=1 / 2 \mathrm{HZ} ; 3=3 / 4 \mathrm{H} ; 5=5 / 8 \mathrm{H} ; 7=7 / 8 \mathrm{H}$.

R. Bras. Zootec., v.31, n.3, p.1362-1366, 2002 (suplemento) dos na Tabela 2, enquanto as médias estimadas do período de serviço, e respectivos erros padrão, para esses mesmos grupos, constam da Tabela 3. A média geral encontrada para o período de serviço foi de 116,8 dias, valor próximo ao citado por Freitas et al. (1997) que encontraram valor de 135 dias quando analisaram um total de 1627 observações, dessa mesma variável, em um rebanho de vacas mestiças. O nível de manejo adotado pelos rebanhos usados neste estudo, que inclui desde a formulação de dietas nas propriedades, controle reprodutivo, produtivo e sanitário adequados, sem dúvida contribuiu favoravelmente sobre a maior parte dos índices técnicos obtidos.

Com referência à análise da produção total de leite, duração da lactação e produção de leite por dia de intervalo de partos (Tabela 4), verificou-se que a maior parte dos fatores de variação, incluídos no

Tabela 3 - Médias estimadas do período de serviço (dias), erros-padrão (EP) e número de observações $(\mathrm{N})$, dos grupos genéticos

Table 3 - Estimated means for service length (days), standard error (SE) and number of observations (N), according to genetic groups

\begin{tabular}{lccc}
\hline Grupo genético & $\mathrm{N}$ & Média & $\mathrm{EP}$ \\
Genetic group & $N$ & Mean & $S D$ \\
\hline 1 & 633 & 94,7 & $\pm 2,63$ \\
3 & 543 & 121,9 & $\pm 2,81$ \\
5 & 98 & 141,3 & $\pm 6,55$ \\
7 & 98 & 120,2 & $\pm 6,55$ \\
\hline
\end{tabular}

$1=1 / 2 \mathrm{HZ} ; 3=3 / 4 \mathrm{H} ; 5=5 / 8 \mathrm{H} ; 7=7 / 8 \mathrm{H}$. 
Tabela 4 - Síntese da análise de variância para as características produtivas

Table 4 - Variance analyses for productive traits

\begin{tabular}{lccc}
\hline $\begin{array}{l}\text { Variável } \\
\text { Variable }\end{array}$ & PT(1) & DL(2) & PLIEP(3) \\
RL(2) & $M Y C I(3)$ \\
\hline $\begin{array}{l}\text { Rebanho } \\
\text { Ano do parto }\end{array}$ & $293805991^{* *}$ & $310149,6^{* *}$ & $1826,7^{* *}$ \\
$\begin{array}{l}\text { Year of calving } \\
\text { Grupo genético }\end{array}$ & $46449028^{* *}$ & $49218,7^{* *}$ & $121,1^{* *}$ \\
$\begin{array}{l}\text { Genetic group } \\
\text { Estação do parto } \\
\text { Season of calving }\end{array}$ & $3293350^{\mathrm{NS}}$ & $3073,3^{\mathrm{NS}}$ & $25,7^{\mathrm{NS}}$ \\
$\begin{array}{l}\text { Ordem do parto } \\
\text { Calving order }\end{array}$ & $26112713^{* *}$ & $10411,4^{*}$ & $95,8^{* *}$ \\
\hline
\end{tabular}

NS = não significativo; ${ }^{*}(p<0,01) ;{ }^{*}(p<0,05)$.

$(1)=$ produção total de leite, $(2)=$ duração da lactação, $(3)=$ produção de leite por dia de intervalo de partos.

$N S=$ not significant; ${ }^{* *}(p<0.01) ;{ }^{*}(p<.05)$.

(1)= milk yield ; (2) lactation length; (3) = milk yield per day of calving interval.

modelo de análise, foi significativa, exceto estação do parto, indicando que essas características foram fortemente influenciadas pelos mesmos. A média observada da produção total de leite foi de $4180,8 \mathrm{~kg}$, com desvio padrão de $1824 \mathrm{~kg}$, enquanto que, para cada grupo genético, os valores foram os seguintes: 3434,9 $\mathrm{kg} ; 4569,5 \mathrm{~kg} ; 4533,1 \mathrm{~kg}$; e $5470,0 \mathrm{~kg}$, respectivamente, para os grupos $1 / 2 \mathrm{HZ}, 3 / 4 \mathrm{H}, 5 / 8 \mathrm{H}$ e $7 / 8 \mathrm{H}$. Analisando essas médias das produções de leite por grupo genético, nota-se que o grupo $7 / 8 \mathrm{H}$ foi superior aos demais com relação à produção, embora deva ser considerado que esses animais são aqueles que apresentam maior proporção de genes da raça Holandesa, devido ao cruzamento absorvente que vem sendo praticado pela propriedade. Outros fatores devem ter contribuído para o aumento da produção de leite no rebanho no decorrer dos anos, como as modernas práticas de alimentação e manejo que vêm sendo oferecidas aos rebanhos nos últimos anos, somados ao uso de reprodutores de melhor potencial genético de produção.

$\mathrm{Na}$ análise dos parâmetros genéticos, os valores de herdabilidades $\left(\mathrm{h}^{2}\right)$ estimados, para a maioria das características reprodutivas (Tabela 5), foram em geral próximos de zero, concordando com vários citações da literatura como Grosshans et al. (1997); Monardes et al. (1995); Silva et al. (1998) e Balieiro et al.(1997), que confirmam serem essas características predominantemente condicionadas e dependentes dos fatores ambientais, com exceção do valor da $\mathrm{h}^{2}$
Tabela 5 - Estimativas de herdabilidades $\left(\mathrm{h}^{2}\right)$, errospadrão (EP) e número de observações $(N)$ para as características reprodutivas

Table 5 - Heritability estimates $\left(h^{2}\right)$, standard error (SE) and number of observations $(N)$ for reproductive traits

\begin{tabular}{lccc}
\hline Característica & $\mathrm{N}$ & $\mathrm{h}^{2}$ & $\mathrm{EP}$ \\
Trait & $N$ & & $S D$ \\
\hline IPC & 806 & 0,31 & $\pm 0,13$ \\
$A F C$ & & & \\
PP1S & 1370 & 0,04 & $\pm 0,04$ \\
ICFS & & & $\pm 0,03$ \\
PS & 1370 & 0,00 & $\pm 0,04$ \\
$D O$ & 1433 & 0,02 & \\
IEP & & & \\
$C I$ & &
\end{tabular}

$\mathrm{IPC}=$ idade à primeira cria; $\mathrm{PP} 1 \mathrm{~S}=$ período parto primeiro serviço; $\mathrm{PS}=$ período de serviço; IEP = intervalo de partos.

$A F C=$ age at first calving; ICFS = interval from calving to first service; $D O=$ days open; $\mathrm{Cl}=$ calving interval.

para a IPC que se pode considerar mediano, 0,31 , ao contrário de alguns resultados mostrados pela literatura. As estimativas das correlações genéticas entre IEP e PS e entre PP1S e IEP foram altas e próximas da unidade, o oposto dos valores encontrados por Grosshans et al. (1997) e Silva et al. (1992). Enquanto a produção total de leite, duração da lactação e produção de leite por dia de intervalo de parto mostraram valores de herdabilidade de 0,28; 0,05 e 0,10, respectivamente (Tabela 6), situando-se próximos dos resultados citados por Balieiro et al. (1997) e Zambianchi et al. (1997). Os valores das correlações genéticas entre a produção de leite e intervalo de

Tabela 6 - Estimativas dos componentes de variância, de herdabilidade $\left(\mathrm{h}^{2}\right)$ e erros padrão (EP)

Table 6 - Estimates of components of variance, heritability $\left(h^{2}\right)$ and standard error (SE). by productive traits

\begin{tabular}{lcccc}
\hline $\begin{array}{l}\text { Característica } \\
\text { Trait }\end{array}$ & $\mathrm{h}^{2}$ & $\sigma_{\mathrm{a}}^{2}$ & $\sigma_{\mathrm{e}}^{2}$ & $\mathrm{EP}$ \\
\hline PT & 0,20 & 500812,07 & 1022737,65 & $\pm 0,082$ \\
$M Y$ & 0,07 & 214,86 & 2986,22 & $\pm 0,057$ \\
DL & & & & \\
LL & 0,06 & 1,39 & 9.29 & $\pm 0,096$ \\
PLIEP & & & & \\
MYCI & & & &
\end{tabular}

$\left(\sigma^{2}\right)$ = variância genética aditiva, $\left(\sigma^{2}\right.$ e $)=$ variância residual, $\mathrm{PT} \stackrel{\mathrm{a}}{=}$ produção total de leite; $\mathrm{DL}=$ duração da lactação; PLIEP= produção de leite por dia de intervalo de parto.

$\left(\sigma^{2}{ }_{2}\right)=$ additive genetic variance; $\left(\sigma^{2}{ }_{e}\right)=$ residual variance; $M Y=$ milk yield; $L L=$ lactation length; $\mathrm{MYCl}=$ milk yield per day of calving interval. 
partos ficaram próximos da unidade, devendo ser vistos com certa cautela, devido ao restrito número de informações. Para a correlação entre a produção de leite e duração da lactação encontrada foi de 0,81 , podendo ser considerada de alta magnitude.

\section{Conclusões}

Os resultados deste estudo permitem concluir que em rebanhos leiteiros comerciais bem manejados, é possível obter índices reprodutivos satisfatórios associados a bons níveis de produção de leite. Sobre os valores das herdabilidades das características reprodutivas, os resultados confirmam que as mesmas dependem em grande parte do manejo oferecido aos rebanhos. Com referência ao valor de herdabilidade, obtido para produção de leite ficou confirmado que essa característica apresenta variância genética aditiva média, podendo constituir valioso auxílio na seleção.

\section{Literatura Citada}

BALIEIRO, J.C.C.; MILAGRES, J.C.; BALIEIRO, E. S. et al. Aspectos genéticos e fenotípicos em características reprodutivas do rebanho leiteiro da Universidade Federal de Viçosa. In: REUNIÃO DA SOCIEDADE BRASILEIRA DE ZOOTECNIA, 34., 1997. Juiz de Fora. Anais... Juiz de Fora: Sociedade Brasileira de Zootecnia, 1997. p.121-123.

BOLDMAN, K.G.; KRIESE, L.A., Van VLECK, L.D. A manual for use for MTDFREML: a set of programs to obtain estimates of variance and covariances [DRAFT]. Lincoln: Department of Agriculture. Agricultural Research Service, 1995. $114 p$.

FERREIRA, J.J.; FERREIRA, M.B.D. Sistema de Produção de Leite da EPAMIG - Desempenho por grupo racial e custo de produção de leite. In: ENCONTRO DE PRODUTORES DE GADO LEITEIRO F1, 2., 1998. Belo Horizonte. Anais... Belo Horizonte: 1998. p.19-28.

FERREIRA, J.J.; MADALENA, F.E. Efeito do sistema de cruzamento sobre o desempenho produtivo e reprodutivo de vacas leiteiras. Arquivo Brasileiro de Medicina Veterinária e Zootecnia, v.49, n.6, p.74-75, 1997.

FREITAS, A.F.; TEIXEIRA, N.M.; VALENTE, J. et al - Fatores genéticos e de ambiente sobre características produtivas e reprodutivas em rebanhos de animais mestiços. In: REUNIÃO ANUAL DA SOCIEDADE BRASILEIRA DE ZOOTECNIA, 33., 1996, Fortaleza. Anais... Fortaleza: Sociedade Brasileira de Zootecnia 1996. p.59-60.
FREITAS, M.A.R.; GUILHERMINO, M.M.; ZAMBIANCHI, A.R. et al. Monitoring Brazilian Dairy Herds: Past, Present and Future. In: EAAP MEETING, 48., 1997, Vienna. Papers...Viena: 1997. p.286.

GROSSHANS, T.; XU, Z.Z.; BURTON, L.J. et al. Performance and genetic parameters for fertility of seasonal dairy cows in New Zealand. Livestock Production Science, v.51, p.41-51, 1997.

MADALENA, F.E.; LEMOS, A.M.; TEODORO, R.L. et al. Desempenho comparativo de vacas mestiças Holandês Guzerá de origem leiteira e não leiteira. In: REUNIÃO ANUAL DA SOCIEDADE BRASILEIRA DE ZOOTECNIA, 33., 1996, Fortaleza. Anais... Fortaleza: Sociedade Brasileira de Zootecnia, 1996. p.3-4.

MONARDES, H.G; ALMEIDA, R.; RIBAS, N.P. Estudo da idade ao primeiro parto em vacas da raça holandesa, Região Batavo, Paraná. In: REUNIÃO ANUAL DA SOCIEDADE BRASILEIRA DE ZOOTECNIA, 32., 1995, Brasília. Anais... Brasília: Sociedade Brasileira de Zootecnia, 1995. p.688-689.

PRADO, E.; CRUZ, F.E.R.; VIANNA, A.M.C. et al. Avaliação do desempenho técnico econômico de exploração leiteira em Divinópolis. Arquivo Brasileiro de Medicina Veterinária e Zootecnia, v.47, p.361-372, 1995.

STATISTICAL ANALISYS SYSTEM INSTITUTE - SAS. User's guide: Statistics. Cary: 1992.

SILVA,H.M.; WILCOX, C.J.; THATCHER, W.W. et al. Factors affecting days open, gestation length, and calving interval in Florida dairy cattle. Journal of Dairy Science, v.75, p.28823, 1992.

SILVA, M.V.G.B.; BERGMANN, J.A.G.; MARTINEZ, M.L. et al. Associação genética, fenotípica e de ambiente entre medidas de eficiência reprodutiva e produção de leite na raça Holandesa. Revista da Sociedade Brasileira de Zootecnia, v.27, n.6, p.1115-1122, 1998.

ZAMBIANCHI, A.R.; FREITAS, M.A.R.; PEREIRA, C.S. Aspectos genéticos e de ambiente da produção de leite e intervalo entre partos de vacas da raça holandesa. In: REUNIÃO ANUAL DA SOCIEDADE BRASILEIRA DE ZOOTECNIA, 34., 1997, Juiz de Fora. Anais... Juiz de Fora: Sociedade Brasileira de Zootecnia, 1997. p.13-15. 